\title{
Periapical radiographic technique errors made with digital sensors
}

\author{
Victória Conterato Dias ${ }^{1}$, Aline Rose Cantarelli Morosolli,", ${ }^{2,}$ Maria Ivete Bolzan Rockenbach ${ }^{3}$ \\ ${ }^{1}$ Graduation Student, ${ }^{2,3}$ Professor, Dentistry Degree Program, School of Health Science, Pontifical Catholic University of Rio \\ Grande do Sul, Porto Alegre, Brazil
}

*Corresponding Author:

Email: ivete.rockenbach@pucrs.br

\begin{abstract}
Purpose: The objective of this study was to analyze the errors of technique in periapical radiographs as performed by fourth year students at the Dentistry School of the Pontifical Catholic University of Rio Grande do Sul, Brazil.

Material and Methods: Fifteen students were randomly selected and each one performed a complete periapical examination in a mannequin. A Direct Digital X-Ray System equipped with a size 2 Sensor was used to obtain the radiographic images. A total of 210 periapical radiographs were evaluated and the images were classified according to the errors that were related to the radiographic technique. The students were also asked to report on their experience with the direct digital system.

Results: The absence of a safety margin was the most frequent error on the radiographs that were performed by the students, while elongated image errors were not found. Regarding the radiographed regions, the highest frequency of errors was found in the upper canine region on the left side.

Conclusion: The need for a greater training of the students of dentistry, in order to better perform the radiographic technique and to decrease the occurrence of technique errors in periapical radiographs when using a digital receptor, is emphasized.
\end{abstract}

Keywords: Digital radiography, Diagnostic imaging, Learning, Students.

\section{Introduction}

The radiographic examination is an auxiliary diagnostic tool that is used in dental clinic procedures. It provides important data for a completion of the information which is obtained in the clinical examination. Radiographs play a fundamental role in the analysis and the evaluation of the teeth and jaws. ${ }^{1}$ Periapical radiography is usually performed by the bisector technique. The purpose of this radiographic exam is to obtain a view of the whole tooth, including the surrounding alveolar bone. ${ }^{2}$

The acquisition of radiographic images is in a phase of transition, in which the conventional radiographic method, which uses radiographic films as image receptors, is gradually being replaced by digital systems that have as receptors of images, phosphor plates or sensors. Undergraduate dental students receive an initial training in many schools when using radiographic films. As the new systems become available, these students begin to perform the radiographic technique with digital receptors and apply as a base, the knowledge that was acquired with the use of radiographic films.

The correct execution of the radiographic technique has the purpose of obtaining an ideal image for the accomplishment of an accurate diagnosis. Due to the occurrence of technique errors during an X-ray image receptor exposure, it is important to identify those errors that occur more frequently. Thus, targets can be set in order to decrease or prevent these occurring and, above all, to propose and to protect the patients from unnecessary ionizing radiation.

The aim of this study was to analyze the performance of the periapical radiographic technique, as performed by undergraduate students of dentistry, when using digital receptors instead of radiographic films. For this, the errors in the periapical radiographs, referring to the radiographic technique itself, were identified and quantified. The students' experience in relation to the direct digital system was also evaluated and compared with the conventional method.

\section{Material and Methods}

The present research was inserted into a quantitative paradigm with a comparative approach, relating the errors of radiographic technique to the regions in which they occurred with a greater frequency. Qualitative analyzes of the participants' reports were also performed on the execution of the radiographic technique with a sensor type image receptor. This study was approved by the Scientific and Ethics Committee of the Dentistry School of the Pontifical Catholic University of Rio Grande do Sul PUCRS, Brazil (Protocol\# 1.639.746).

Fifteen (15) dental students in their fourth year at the Dentistry School of the Pontifical Catholic University of Rio Grande do Sul (PUCRS), Brazil, were randomly selected for the execution of the periapical radiographic technique. All of the study participants signed the informed consent form. These students received a numbering for the later analyzes of the data. The radiographs were performed in a mannequin.

The periapical digital images were acquired by using a FONA CDR Elite Direct Digital X-Ray System equipped with a size 2 Sensor powered by Schick (Sirona Dental, Inc., Long Island City, New York, USA). This receptor has a CMOS-APS (complementary metal oxide semiconductor active pixel sensor) imager, 
with an external area of $30 \times 43 \mathrm{~mm}^{2}$, an active area of $26 \times 36.5 \mathrm{~mm}^{2}$ and a resolution of $27 \mathrm{ppl} / \mathrm{mm}^{2}$ (line pairs per square millimeter). The image processing and analyzes were performed by using the digital system's specific software.

In order to select the exposure time, as well as the electrical factors of the X-ray apparatus, the area to be radiographed, and the image receptor used, these items were all independently assessed and noted. The X-ray apparatus that was used was a Sommo (Gnatus, Ribeirão Preto, SP, Brazil), with an electric regime of $70 \mathrm{kVp}, 7 \mathrm{~mA}$, and a total filtration of $3.22 \mathrm{~mm}$ of aluminum. For the realization of the radiographic technique, positioners that were suitable for the sensors were also used (Indusbello, Londrina, Paraná, Brazil).

Each student performed a complete periapical examination, with radiographs that were considered to be technically correct. The exposures included the regions of the incisors, the canines, the premolars, and the molar regions, in both the upper and lower jaws. The students performed the expositions until they considered that the images were ideal for a radiographic diagnosis. Therefore, repetitions were performed, as often as deemed necessary.

The digital radiographs were evaluated by a properly calibrated observer. The images were analyzed individually in an environment with controlled lighting, through a monitor with physical characteristics, according to the visualization needs.

The images were classified according to the identified errors that were related to the radiographic technique, such as:

a) Incorrect vertical angulation: shortened or elongated image;

b) Incorrect horizontal angulation: beam of the X-ray not parallel to the proximal surfaces of the teeth;

c) Incorrect incidence point: cone cutting image;

d) Incorrect positioning of the receptor (an excessive or the absence of a safety margin; the safety margin not being parallel to the incisal or occlusal surface; an incorrect long axis of the receptor; a poor receptor centering).
After the digital radiography was performed, the students were asked to:

1. Describe your experience when using the direct digital radiographic system (with a sensor) and compare it with your previous experience of the conventional method (radiographic film);

2. Report on the advantages and the disadvantages and / or the limitations in the use of the digital system, when compared to the conventional radiographic method.

Statistical analyzes of this study were performed through descriptive statistics and comparisons between the groups. In order to evaluate the intra-rater reliability, the Kappa test was used.

The data that was obtained was tabulated and the percentages were calculated, while considering the frequency of occurrence of the radiographic technique errors. The qualitative data was reported by using percentages. The Mann-Whitney statistical test was used in order to correlate the presence of errors with the number of X-ray repetitions. In addition, in order to verify if there was a correlation between the number of errors and the number of repetitions, Spearman's Correlation Coefficient was applied. The results were considered statistically significant at 0.05 .

\section{Results}

The intra-rater reliability that was verified by using the Kappa test showed a variation of between $73.7 \%$ and $99 \%$. Thus, we opted to perform new analyzes of the radiographic images, so that the final results that were obtained accorded with the most prevalent agreement among the three analyzes.

A total of 210 periapical radiographs were analyzed. It was observed that $92.8 \%$ of the radiographs presented some type of a radiographic technique error. The most frequent error was the absence of a safety margin $(51.9 \%)$, followed by a poor receptor centering $(38.9 \%)$. Among the errors that presented the lowest percentages were: an incorrect long axis of the receptor $(10.5 \%)$; an overlapping of the proximal surfaces of the teeth $(9.5 \%)$; and a cone cutting image $(2.9 \%)$. No elongated image errors were observed (Table 1).

Table 1: Distribution of the types of technique errors observed in periapical radiographs performed by students of the Course of Dentistry of the PUCRS

\begin{tabular}{|l|c|c|}
\hline \multicolumn{1}{|c|}{ Errors } & General & Frequency \\
\cline { 2 - 3 } & $\mathrm{n}$ & $(\%)$ \\
\hline Elongated Image & 0 & 0 \\
\hline Shortened Image & 37 & 17.6 \\
\hline $\begin{array}{l}\text { X-Ray Not Parallel to the Proximal Surfaces } \\
\text { of the Teeth }\end{array}$ & 20 & 9.5 \\
\hline Cone Cutting & 6 & 2.9 \\
\hline Absence of Safety Margin & 109 & 51.9 \\
\hline Excessive Safety Margin & 40 & 19.0 \\
\hline Non-Parallel Safety Margins & 60 & 28.6 \\
\hline
\end{tabular}




\begin{tabular}{|l|l|l|}
\hline Poor Receptor Centring & 81 & 38.6 \\
\hline Incorrect Long Axis of the Receptor & 22 & 10.5 \\
\hline
\end{tabular}

Table 2 shows the distribution of the frequency of the technique errors that were observed in the periapical radiographs, in the different regions of the maxilla and the mandible.

Table 2: Distribution of the frequency of errors, according to the regions, observed in periapical radiographs performed by students of the Course of Dentistry of the PUCRS

\begin{tabular}{|l|c|c|c|c|c|c|c|c|}
\hline & $\begin{array}{c}\text { Shortene } \\
\text { d } \\
\text { Image }\end{array}$ & $\begin{array}{c}\text { X-Ray Not } \\
\text { Parallel to } \\
\text { the Proximal } \\
\text { Surfaces of } \\
\text { the Teeth }\end{array}$ & $\begin{array}{c}\text { Cone } \\
\text { Cutting }\end{array}$ & $\begin{array}{c}\text { Absence of } \\
\text { Safety } \\
\text { Margin }\end{array}$ & $\begin{array}{c}\text { Excessive } \\
\text { Safety } \\
\text { Margin }\end{array}$ & $\begin{array}{c}\text { Non- } \\
\text { Parallel } \\
\text { Safety } \\
\text { Margins }\end{array}$ & $\begin{array}{c}\text { Poor } \\
\text { Receptor } \\
\text { Centring }\end{array}$ & $\begin{array}{c}\text { Incorrect } \\
\text { Long Axis } \\
\text { of the } \\
\text { Receptor }\end{array}$ \\
\cline { 2 - 9 } & $\mathrm{n}(\%)$ & $\mathrm{n}(\%)$ & $\mathrm{n}(\%)$ & $\mathrm{n}(\%)$ & $\mathrm{n}(\%)$ & $\mathrm{n}(\%)$ & $\mathrm{n}(\%)$ & $\mathrm{n}(\%)$ \\
\hline UI & $2(13.3)$ & - & - & $13(86.7)$ & - & - & $13(86.7)$ & - \\
\hline LI & $6(40.0)$ & - & $1(6.7)$ & $11(73.3)$ & $1(6.7)$ & $1(6.7)$ & $11(73.3)$ & - \\
\hline RUC & $2(13.3)$ & $9(60.0)$ & - & $13(86.7)$ & - & - & $5(33.3)$ & $1(6.7)$ \\
\hline LUC & $9(60.0)$ & $9(60.0)$ & $6(40.0)$ & $11(73.3)$ & $1(6.7)$ & - & $3(20.0)$ & $1(6.7)$ \\
\hline RLC & $10(66.7)$ & - & $1(6.7)$ & $13(86.7)$ & - & - & $6(40.0)$ & - \\
\hline LLC & $7(46.7)$ & - & - & $11(73.3)$ & - & - & $1(6.7)$ & - \\
\hline RUPM & - & - & $1(6.7)$ & $3(20.0)$ & $1(6.7)$ & $11(73.3)$ & - & $3(20.0)$ \\
\hline LLPM & - & $1(6.7)$ & & $1(6.7)$ & $9(60.0)$ & $8(53.3)$ & - & $2(13.3)$ \\
\hline RLPM & $1(6.7)$ & - & - & $1(6.7)$ & $9(60.0)$ & $15(100.0)$ & $2(13.3)$ & $2(13.3)$ \\
\hline LLPM & - & - & - & $7(46.7)$ & $2(13.3)$ & $10(66.7)$ & $1(6.7)$ & $3(20.0)$ \\
\hline RUM & - & $1(6.7)$ & - & $10(66.7)$ & $1(6.7)$ & $2(13.3)$ & $10(66.7)$ & $3(20.0)$ \\
\hline LUM & - & - & - & $2(13.3)$ & $9(60.0)$ & $3(20.0)$ & $6(40.0)$ & $2(13.3)$ \\
\hline LLM & - & - & - & $3(20.0)$ & $4(26.7)$ & $4(26.7)$ & $10(66.7)$ & $2(13.3)$ \\
\hline LLM & - & - & $2(13.3)$ & $10(66.7)$ & $3(20.0)$ & $6(40.0)$ & $13(86.7)$ & $3(20.0)$ \\
\hline
\end{tabular}

UI: Upper Incisors

LI: Lower Incisors

RUC: Right Upper Canine

LUC: Left Upper Canine

RLC: Right Lower Canine
LLC: Left Lower Canine

RUPM: Right Upper Premolars

LLPM: Left Lower Premolars

RLPM: Right Lower Premolars

LLPM: Left Lower Premolars
RUM: Right Upper Molars

LUM: Left Upper Molars

LLM: Right Lower Molars

LLM: Left Lower Molars
The most prevalent error was the absence of a margin of safety. This was observed in all of the regions of the maxilla and the mandible, with the upper incisors, the upper and lower canines, both on the right side, being those regions that indicated the highest values $(86.7 \%)$. The regions of the upper premolars on the left and on the lower sides on the right side presented the lowest indices for this error, both with $6.7 \%$. The second most noticed type of error was of a poor sensor centering, with the highest index being in the upper incisors and the left lower molar regions, both with $86.7 \%$. While the lowest index for this error (6.7\%) was found in the canines and in the lower premolar regions on the left side. The regions of the upper and right premolars presented no errors of poor receptor centering.

In relation to the distribution of the technique errors by the X-ray regions, the highest frequency was found in the upper left canine region. In this region, the most frequent errors were the absence of a safety margin $(73.3 \%)$, followed by a shortened image and the $\mathrm{X}$-ray not being parallel to the proximal surfaces of the teeth, both with $60 \%$. A percentage of $100 \%$ for the margin of a safety error not being parallel to the occlusal surface in the region of the lower right premolars was evidenced. This was while at the same time, elongated image errors were not found in the radiographs that were performed by the students.

The frequency of an error that was less committed by the students, when considering the X-ray regions, was a percentage of $6.7 \%$ that was found for the mistake of the X-ray not being parallel to the proximal surfaces of the teeth in the regions of the upper premolars and the right upper molars. It was also evidenced that there was a percentage of $6.7 \%$ for an image error with a cone cutting in the right lower canine region, the lower incisors, and the right upper premolars. For an incorrect long axis error of the receptor, the highest prevalence was observed in the posterior regions of the maxilla and the mandible, whereas in the regions of the upper canines on the right and left sides, only minor frequencies for this error were found.

The Mann-Whitney statistical test was used in order to correlate the presence of errors with the number of repetitions of the radiographs. It was observed that the error of a poor receptor centering presented a statistically significant value $(\mathrm{p}=0.028)$.

In relation to the distribution of the number of students who performed the repetitions, it was possible to observe that all of the students repeated the radiography of the lower premolar region on the right 
side, persisting with the margin of a safety error not being parallel to the occlusal surface. In all of the regions of the maxilla and the mandible, there was at least one student who repeated the radiography because of some kind of an error. The shortened image error showed a statistically significant value for the lower left canine region $(0.036 \%)$, in which 10 students performed repetitions.

In order to verify the correlation between the number of errors and the number of repetitions, Spearman's Correlation Coefficient was used, and it was observed that there was no positive correlation (coefficient $\mathrm{r}=0.61 ; \mathrm{P}=0.376$ ).

Table 3: Correlation between the number of errors and the number of repetitions of periapical radiographs performed by students of the Course of Dentistry of the PUCRS

\begin{tabular}{|l|l|c|c|}
\hline \multicolumn{2}{|l|}{} & Repetition & Number of errors \\
\hline Repetition & Correlation coefficient & 1.000 & 0.061 \\
\hline & Sig. (2-talied) & - & 0.376 \\
\hline & $\mathrm{N}$ & 210 & 210 \\
\hline $\begin{array}{l}\text { Number of } \\
\text { errors }\end{array}$ & Correlation coefficient & 0.061 & 1.000 \\
\hline & Sig. (2-talied) & 0.376 & - \\
\hline & $\mathrm{N}$ & 210 & 210 \\
\hline
\end{tabular}

The results of the analyzes of the students' experience when using the direct digital radiographic system (with sensor), when compared with the conventional method (radiographic film), found that $67 \%$ of the students mentioned that the digital system had a faster acquisition of the image; while $53 \%$ of the students reported a difficulty in positioning the sensor due to its rigidity; and $26 \%$ of the students reported a difficulty in positioning for the anterior regions. On the other hand, $13 \%$ of the students described that they preferred to use the conventional method, but they emphasized the practicality of the digital system, due to the easier positioning of the sensor in the posterior regions. However, they also reported that the greater number of repetitions was due to the difficulty of positioning the sensor. For $7 \%$ of the students, the degree of difficulty in using the digital system was the same as it was for the conventional method. In addition, $7 \%$ of the students emphasized the facility of the digital system, the elimination of the processing solutions, the digital storage of the images, as well as the training necessity of using the digital system with sensors, due to its limitations in relation to its size and its rigidity.

The students' opinion about the advantages of using the digital system, when compared to the conventional radiographic method, revealed that $87 \%$ of the students reported on the speed to obtain the image; 53\% described the reduction of exposure time; and $20 \%$ who spent less clinical time. On the other hand, $7 \%$ of the students reported that the use of the digital system was simpler, it had a higher accuracy and it produced a higher quality. They considered that the storage being managed in computers, the elimination of the darkroom, and the possibility of a manipulation of the image, the radiographic technique had less of a probability of errors.

When in relation to the disadvantages, $53 \%$ of the students considered that there was a difficulty in positioning due to the sensor's rigidity; $33 \%$ reported that the sensor wire interfered with their positioning; and $26 \%$ reported a greater number of repetitions. Whereas, $13 \%$ described a greater discomfort for the patient, a lack of flexibility, the sensor size and a difficulty in performing the technique in patients with a small mouth opening. Moreover, $7 \%$ of the students reported on the high cost, a difficulty of positioning in the anterior regions due to the curvature of the palate, a difficulty in obtaining a correct margin of safety, the handling of a computer, and the greater clinical time.

\section{Discussion}

During the exposure of the image receptors to Xrays, errors may occur due to the execution of the radiographic technique. The purpose of this study has been to evaluate the errors that occurred more frequently, since by identifying them, it would be possible to improve the performance of the technique and the teaching of Dental Radiology. On the other hand, the identification of these errors has been an indicator of quality and quality becomes an important tool in the perception of those areas that require the optimization of an image for an accurate interpretation. In addition, repeated examinations contribute to an increment in the dose of radiation to the patient, whilst at the same time, they become an inconvenience to them. Furthermore, there is an increase in the time spent by the professional who is working with the $\mathrm{X}$ rays. What is more, there is a wearing down and a deterioration of the equipment and accessories.

Haghnegahdar et $\mathrm{al}^{3}{ }^{3}$ evaluated 3188 radiographs, of which 113 were classified as unacceptable and 181 presented some type of error. In the present study, it was observed that in 195 (92.8\%) of the radiographs, there was some kind of radiographic technique error.

The most frequent technical errors were those which related to the positioning of the image receptor, among which there was no safety margin (51.9\%); followed by a poor receptor centering (38.6\%); together 
with a non-parallel safety margin to the incisal or occlusal surface $(28.6 \%)$. Haghnegahdar et al. ${ }^{3}$ performed a study during the period of one academic semester, in which $35.4 \%$ of the radiographs had an incorrect positioning of the receptor, while in that same study, the highest frequency of errors occurred in the region of the upper molars. Elangovan et al., ${ }^{4}$ in a research with undergraduate students, obtained an incorrect sensor positioning in $26.7 \%$ of the radiographs.

In this study, the absence of a margin of safety was the most prevalent error. This error can be due to the difficulty of the students to correctly use the positioner and / or also be due to the rigidity of the sensor.

An elongated image error was not found in the present study, however, a shortened image error with a frequency of $17.6 \%$ was observed. This may have occurred due to the difficulty of a correct positioning of the sensor in the positioner. In a study conducted by Viero et al., ${ }^{5}$ when evaluating a total of 168 radiographs that were performed with phosphor plates and circular cylinder collimators, $90.6 \%$ of errors were presented and a percentage of 0.6 was found for the elongated image error in the periapical radiographs that were performed by the undergraduate students. Moreover, Masserat et al. ${ }^{6}$ observed that $94.9 \%$ of the periapical radiographs made by the students of the 7 th semester presented a technique error, with the highest frequency being for an elongated image (19\%).

Haghnegahdar et al. ${ }^{3}$ found that the elongated image error was twice as large as the shortened image error. For the same authors, a distribution of the technique errors by X-ray regions was higher in the region of the maxillary molars. The results in the present study have shown that the highest frequency of errors was in the upper left canine region and the lowest frequency was in the left lower canine and the upper right premolar regions.

In another study, the occurrence of radiographic errors was higher when sensors were used in comparison to films, due to the smaller area of the active area of the sensor. ${ }^{4}$ As a result, the number of radiographic images that were required for an examination of the same region increased. Due to this factor, the effect of a decrease in the dose of ionizing radiation in systems that use sensors can be questioned. $^{7}$

In the present study, we also considered the number of repetitions that were required in order to obtain an ideal radiograph for a diagnosis. It was observed that all of the students repeated the radiography in the region of the lower premolars on the right side and they persisted with a margin error that was not parallel to the occlusal surface. This error can be justified, since the sensor is stiffer than radiographic film, which consequently generates a difficulty in the correct positioning of the sensor. ${ }^{2}$
The high number of repetitions performed by the students was justified by the fact that the radiographs were carried out on a mannequin, so many students did not take into account the radiation dose for each exposure. Thus, it is possible that the number of repetitions in those patients with an X-ray imaging was lower.

In a study published by Kreich et al., ${ }^{8}$ they analyzed 800 radiographs that were performed according to the bisector technique by the students from the $2 \mathrm{nd}, 3 \mathrm{rd}$, 4th and 5th years at the Dentistry School of the State University of Ponta Grossa (UEPG), Paraná, Brazil. The most commonly encountered error was an incorrect placement of the film (57\%). However, there were no differences in relation to the number of errors made by the students of the different years. These authors concluded that, with the passing of the semesters, the students did not evolve significantly in the execution of the radiographic technique. Peker and Alkurt $^{9}$ considered that the number of errors committed by undergraduate students was high. However, these numbers were decreasing as the course progressed.

White and Pharoah $^{2}$ have highlighted the advantages of digital systems, such as the disposal of the darkroom, film processing equipment, as well as the resulting waste of processing solutions and lead foil backing. In the results of this study, $7 \%$ of the students mentioned these advantages and they also emphasized the ease of manipulation of the digital system.

In relation to the disadvantages in the use of the direct digital system, $53 \%$ of the students described a difficulty of positioning due to the rigidity of the sensor. White and Pharoah ${ }^{1}$ considered that the cable attached to the sensor made it difficult to place the sensor in the oral cavity and in the present study, 33\% of the students reported on the same difficulty.

Radiographic film is flexible, and it has a small thickness. These factors may facilitate the execution of the technique; however, a longer exposure time is required when compared to the sensor, which is rigid and has a greater thickness. Based upon the results that were obtained in the present study, it was observed that there was the need for a greater training of the dental students, in order to better perform the radiographic technique and to decrease the occurrence of technical errors when using sensors.

From the results of this work, it has been possible to verify that there were a high number of errors of the radiographic technique when performed by the dental students while using the direct digital system. The error with the highest occurrence was the absence of a safety margin of the sensor, while the elongated image error was not found. The region with the highest frequency of errors was the upper left canine. 


\section{Conclusion}

It was concluded that, even if the direct digital system presents numerous advantages, it is necessary a greater training of the students of dentistry, in relation to the radiographic technique when using a sensor, in order to reduce the occurrence of these errors and the repetitions.

\section{References}

1. White SC, Pharoah MJ. The evolution and application of the dental maxillofacial imaging modalities. Dent Clin North Am 2008;52:689-705.

2. White SC, Pharoah MJ. Oral radiology; principles and interpretation. 7th ed. St. Louis: Mosby Inc; 2014.

3. Haghnegahdar A, Bronoosh P, Taheri MM, Farjood A. Common intra oral radiographic errors made by dental students. I 2013;2:44-8

4. Elangovan S, Nazargi M, Suman J, Senthil BK, Deepika R. Faulty radiographs: A cross sectional analysis among dental college students in Namakkal District, Tamil Nadu. J Pharm Bioallied Sci 2016; 8:S116-S118.

5. Viero F, Morosolli ARC, Rockenbach MIB. Periapical radiographic technique errors made with film and phosphor plates. J Pharm Biol Sci 2017;5:150-54.

6. Masserat V, Ebrahimi HS, Eil N, Mollashahi J, Naeb M. Evaluation of frequency of periapical radiographic errors in Dental Radiology Department in Zahedan in 20142015. Sch J App Med Sci 2017;5:112-15.

7. Hellén-Halme K, Johansson PM, Hakansson J, Petersson A. Image quality of digital and film radiographs in applications sent to the dental insurance office in Sweden for treatment approval. Swedish Dent J 2004;28:77-84.

8. Kreich EM, Queiroz MGS, Sloniak MC. [Quality control in periapical radiographs obtained in the course of Dentistry of UEPG.] [Article in Portuguese]. UNOPAR Cient Ciênc Biol Saúde 2002;8:33-45.

9. Peker I, Alkurt MT. Evaluation of radiographic errors made by undergraduate dental students in periapical radiography. NY State Dent J 2009;75:45-8. 